\section{Safety of pulmonary function testing: data from 20 years}

\section{ABSTRACT}

Background Pulmonary function testing

(PFT) is a key investigation in the evaluation of individuals with respiratory symptoms; however, the safety of routine and specialised PFT testing has not been reported in a large data set. Using patient safety incident (PSI) records, we aimed to assess risk of PFT and to characterise these events and any associated risk factors.

Methods In this single-centre audit, demographics and PSI data were collected and categorised for PFT performed between 1996 and 2016 and subdivided into cardiopulmonary or non-cardiopulmonary events. The severity of each PSI was rated using the NHS National Patient Safety Agency and any hospital admission reported.

Results There were 119 PSIs reported from $186000 \mathrm{PFT}$; that is, $0.6 \mathrm{PSIs}$ per 1000 tests. Cardiopulmonary PSIs were 3.3 times more likely to occur than non-cardiopulmonary (95\% Cl 2.17 to 5.12). Syncope was the most frequently occurring cardiopulmonary PSI. Cardiopulmonary exercise testing was associated with 2 PSIs per 1000 tests. PSIs necessitating hospital admission and/or emergency department attendance occurred approximately once every 10000 tests and there was no PFT-associated mortality.

Conclusion Routine and specialised PFT is safe for patients, in the context of established screening preparticipation guidelines. In the event of a PSI, these are likely to be low risk of harm. Our findings highlight the most common PSIs encountered during PFT to facilitate risk reduction.

\section{INTRODUCTION}

Pulmonary function testing (PFT) is a key investigation in the evaluation of any individual with respiratory symptoms. It is estimated that approximately one million formal PFTs are performed in the UK annually. ${ }^{1}$

An understanding of patient risk is important when performing any investigation. Recognised potential complications of PFT include syncope, dizziness and bronchospasm. More serious complications include cardiac and vascular events. ${ }^{2-5}$

There is clear guidance available regarding contraindications for spirometry, bronchoprovocation challenge and cardiopulmonary exercise testing (CPET), ${ }^{6}$ and laboratories should have an established screening process to minimise risk. There is currently however no published data regarding the safety of PFT when employing this approach. We evaluated the safety of PFT, reporting experience of the past 20 years, using patient safety incidents (PSIs) to characterise events and associated risk factors.

\section{METHODOLOGY}

A single-centre audit was conducted, using anonymised PSIs, from 1996 to 2016. Clinical PSIs were collated using the NHS Incident Records and/or hospital 'DATIX' system, registered as an audit with Royal Brompton Hospital NHS Foundation Trust.

Each PSI was categorised by type and severity and categorised as cardiopulmonary (eg, pneumothorax, syncope, haemoptysis, chest pain and so on) or non-cardiopulmonary (eg, abdominal pain, fall, hypoglycaemia and so on). Tests were divided into routine PFTs or specialised PFTs (including CPET, hypoxic inhalation challenge, bronchoprovocation and hyperventilation provocation testing (HVPT)). PSI severity was categorised by the NHS National Patient Safety system (www.npsa.nhs.uk), graded on the likelihood of the event reoccurring and its implications. Admission, emergency room assessment or cardiac resuscitation team attendance was recorded.

Differences in demographics and PFT parameters were tested using a MannWhitney $\mathrm{U}$ test for continuous variables and $\chi^{2}$ or Fisher's exact for categorical variables. A $p$ value of $<0.05$ was considered significant and SPSS V.24.0 was used for analysis.

\section{RESULTS}

\section{PSI frequency}

Over the 20-year period, 167016 routine PFTs and 19139 specialised PFTs were performed. There were 119 PSIs $(n=117$ patients) reported, of which $80(67 \%)$ occurred during routine PFT and 39 (33\%) during specialised PFT. Thus, the PSI rate was $0.06 \%$, with the highest recorded year at $0.13 \%$ in 2004 (figure 1).

The majority of PSIs $(n=91,78 \%)$ were classified as cardiopulmonary, with fewer $(n=26,22 \%)$ non-cardiopulmonary events; that is, 0.48 PSIs per 1000 tests compared with 0.22 , respectively. There was a 3.3 times higher risk of having a cardiopulmonary event than a non-cardiopulmonary event (95\% CI 2.17 to 5.12 ).

Of the specialist PFTs, HVPT was associated with the highest PSI frequency at 3.44 per 1000 tests (all cardiopulmonary events) (table 1, figure 2A). CPET was associated with 2 PSIs per 1000 tests.
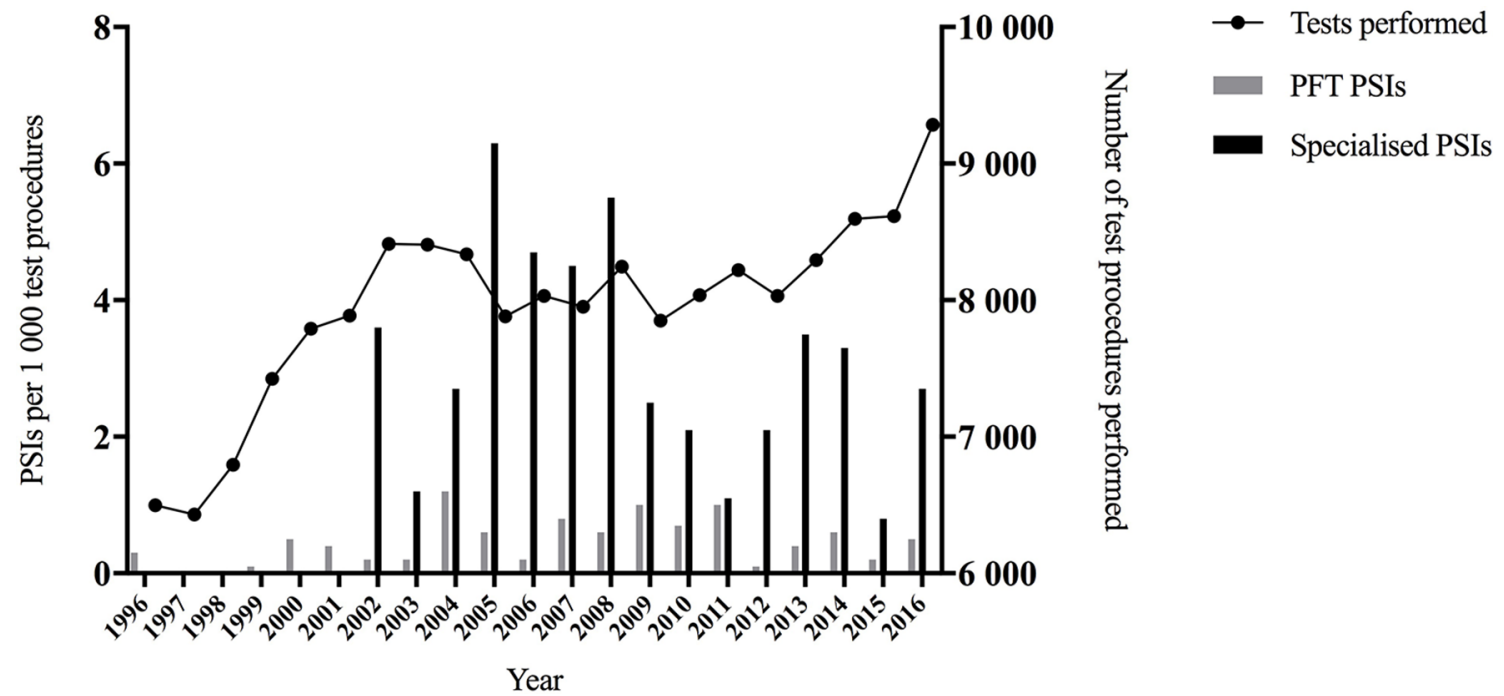

Figure 1 Percentage of PSI in comparison with the number of tests performed. PFT, pulmonary function testing; PSI, patient safety incidents. 


\begin{tabular}{|c|c|c|c|c|c|c|}
\hline & $1996-2000$ & 2001-2005 & $2006-2010$ & 2011-2015 & 2016 & Total \\
\hline HVPT & 0.00 & 5.63 & 8.55 & 0.00 & $-^{*}$ & 3.44 \\
\hline CPET & 0.00 & 1.87 & 4.43 & 5.10 & 0.00 & 2.21 \\
\hline PC20 & 0.00 & 2.11 & 2.72 & 2.89 & 3.72 & 1.88 \\
\hline HIT & 0.00 & 2.14 & 2.54 & 0.00 & 5.73 & 1.55 \\
\hline $\mathrm{pH}$ & 0.00 & 2.06 & 3.11 & 1.27 & 0.00 & 1.40 \\
\hline Routine PFT & 0.09 & 0.61 & 0.70 & 0.46 & 0.54 & 0.48 \\
\hline
\end{tabular}

*No HVPTs were performed in 2016. Note: no incidents were recorded between 1997 and 1998.

CPET, cardiopulmonary exercise test; HIT, hypoxic inhalation test; HVPT, hyperventilation provocation test; PC20, histamine provocation test; pH, oesophageal pH; routine PFT, routine pulmonary function test.

Cardiopulmonary related PSIs were related to younger patients than those with a non-cardiopulmonary PSI $(p=0.02)$, but there was no difference in gender, body mass index (BMI) or smoking status $(\mathrm{p}>0.05)$. Patients who had cardiopulmonary PSIs had a greater transfer factor for carbon monoxide $\%$ predicted $(\mathrm{p}=0.02), \mathrm{FEV}_{1} \%$ predicted $(\mathrm{p}<0.01)$ and $\mathrm{FVC} \%$ predicted $(\mathrm{p}=0.02)$. There was no difference in arterial oxygen saturation between the groups $(p=0.3)$ (table 2).

\section{PSI categories}

The most frequent cardiopulmonary PSI was syncope $(37 \%)$, followed by breathing difficulty (30\%) (figure 2B). Hyperventilation was the most commonly reported cause of breathing difficulty, accounting for 48\% (13 patients) of events. No demographic (eg, age, BMI) or PFT factor allowed differentiation or predicted individuals more likely to have a cardiopulmonary PSI.

\section{PSI severity and outcome}

The majority $(74 \%)$ of reported PSIs were graded as moderate severity. Almost one-fifth of PSIs (23\%) were rated as low severity (ie, unlikely to happen again/ minor consequence). Only one PSI (laryngospasm) was graded severe.

Hospital admission was required for $13 \%$ of all PSIs. The resuscitation team was called to attend on six occasions (5\% of PSIs) and provided clinical input on two occasions. Over the 20-year period,
A

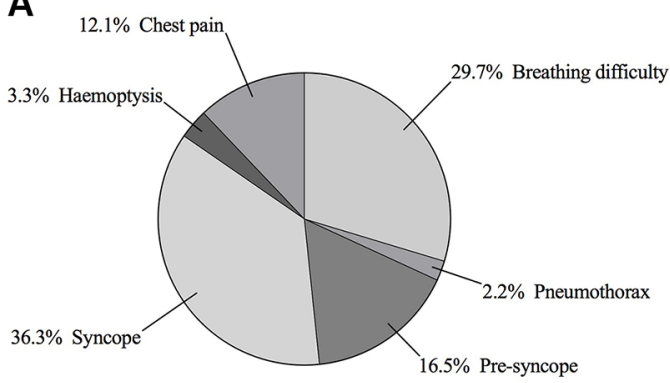

Total $\mathbf{n}=91$

B

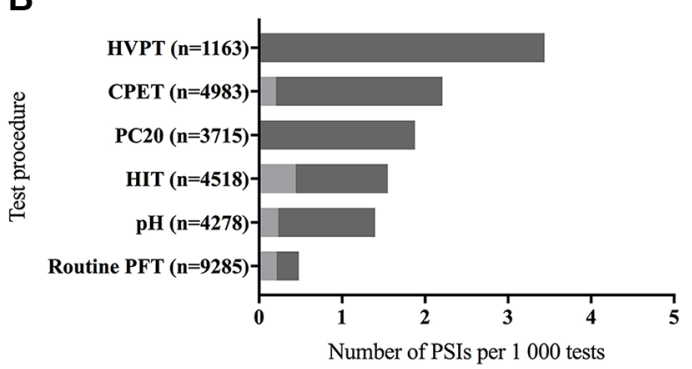

Figure 2 (A) Number of PSIs per 1000 test procedures. (B) Subcategories of recorded cardiopulmonary PSIs. CPET, cardiopulmonary exercise test; HIT, hypoxic inhalation test; HVPT, hyperventilation provocation test; $\mathrm{PC} 20$, histamine provocation test; $\mathrm{pH}$, oesophageal $\mathrm{pH}$; routine PFT, routine pulmonary function test; PSI, patient safety incidents. there was no mortality associated with PFT.

\section{DISCUSSION}

Understanding and characterising the risk of any clinical investigation is important to safeguard patients. Using a widely implemented approach to evaluate and report adverse outcome, we assessed PSI rate in approximately 200000 PFTs and found very few adverse events. Specifically, for every 1000 PFTs performed, less than 1 was associated with a PSI. This compares favourably with other commonly used clinical investigations (eg, phlebotomy-associated syncope (0.26 per 1000)). ${ }^{7}$ When a PSI did occur the majority were classified as moderate risk or less and self-limiting. In addition, PSIs necessitating hospital admission and/or emergency department attendance occurred approximately once every 10000 tests, and resuscitation team input was required on only two occasions over 20 years. Taken together these findings indicate that the patient risk from both routine and specialist PFT is minimal.

Most PFT departments use the American Association for Respiratory Care (AARC) standards contraindications to screen for patient risk during spirometry, bronchoprovocation challenge and CPET. The low level of PSIs we report would support this approach. This acknowledged, PSIs do occur and clearly any PSI is undesirable. In this context it is valuable to study the nature of any PSI and use this information to improve practice (ie, to provide focused training and availability of safety equipment).

Cardiopulmonary PSIs occurred approximately three times more often than non-cardiopulmonary PSIs, with syncope reported as the most common event. This risk can be reduced by modifying PFT in those with a history of situational syncope and by ensuring patients are seated during testing. The second most common subcategory of PSI was 'breathing difficulty'; 
Table 2 Characteristics of subjects with PSIs

\begin{tabular}{|c|c|c|c|}
\hline & Cardiopulmonary & Other & pt \\
\hline $\mathrm{N}$ & 91 & 26 & \\
\hline Gender, female/male & $49 / 42$ & $10 / 16$ & 0.19 \\
\hline Age (years) $\ddagger$ & $49(28)$ & $58(20)$ & $<0.01^{*}$ \\
\hline Body mass index $\left(\mathrm{kg} / \mathrm{m}^{2}\right) \ddagger$ & $26.9(9.5)$ & $25.0(7.8)$ & 0.3 \\
\hline Current or ex-smokers/never-smokers & $32 / 55$ & $11 / 12$ & 0.2 \\
\hline \multicolumn{4}{|l|}{ Pulmonary function } \\
\hline TLCOC \% predicted & $76.5(30.5)$ & $55.9(55.8)$ & $0.02 *$ \\
\hline $\mathrm{FEV}_{1} \%$ predicted $\ddagger$ & $86.1(24.7)$ & $68.8(39.5)$ & $<0.01$ * \\
\hline FVC \% predicted $\ddagger$ & $94.4(23.3)$ & $87.5(38.1)$ & $0.02 *$ \\
\hline $\mathrm{FEV}_{1} / \mathrm{FVC} \neq$ & $75(19)$ & $71(31)$ & 0.4 \\
\hline Resting $\mathrm{SaO}_{2} \neq$ & $96(3)$ & $97(2)$ & 0.3 \\
\hline Resting $\mathrm{PaO}_{2} \ddagger, \mathrm{kPa}$ & $10.9(2.5)$ & $11.9(2.7)$ & 0.3 \\
\hline Resting $\mathrm{PaCO}_{2} \neq, \mathrm{kPa}$ & $5.0(0.9)$ & $5.0(0.9)$ & 0.6 \\
\hline
\end{tabular}

Not all subjects/incidents had all values available.

${ }^{*} \mathrm{p}<0.05$.

tMann-Whitney $\mathrm{U}$ test for continuous variables, and $\chi^{2}$ or Fisher's exact as appropriate for categorical variables.

‡Reported numbers are median (IQR).

PSI, patient safety incident; TLCOC, transfer factor for carbon monoxide when corrected for haemoglobin.

however, the majority of these episodes occurred during HVPT, a test no longer widely used in the UK.

The PSI rate associated with CPET (2 PSIs per 1000 tests) is slightly higher than that reported in a previous studies, ${ }^{8} 0.5$ per 1000 tests and 0.1 events per 1000 tests $^{9}$ (defined as those requiring hospitalisation). The higher PSI rate observed in our series is likely due to the wider PSI criteria employed. Regardless our finding of no serious adverse event from 4983 tests underlines the safety of CPET in the assessment of pulmonary disease.

Our analysis of PSIs has prompted change within the department, including, but not limited to, alterations to the registration system to highlight allergies/prior risk factors, improved staff training for syncope management and alterations to equipment.

Analysis of PSI prevalence is dependent on the quality of data capture, that is, all PSIs being reported in a reliable fashion. It is possible that some PSIs were not 'captured' by our reporting process and thus the data under-represent the true event rate. This acknowledged, the department rigorously advocates PSI recording to progress care. Moreover, the resuscitation team attended on only six occasions over the evaluation period and no deaths were associated with testing.

This data audit was conducted in a specialist tertiary referral centre, which may see more high-risk patients in comparison with other laboratories. The results may thus be associated with a greater overall prevalence of PSIs. Future comparable studies from other centres should be encouraged and may demonstrate a variation across centres.

\section{CONCLUSION}

Routine and specialised PFT is safe for patients, in the context of established screening preparticipation guidelines. In the event of a PSI, these are likely to be low risk of harm. It is unlikely that PFT will ever be entirely without risk; however, by highlighting the most common PSIs encountered, encouraging reporting and acting on adverse events, procedures and local departmental protocols should evolve to further minimise risk.

\section{Cara Roberts, ${ }^{1}$ Simon Ward, ${ }^{1}$ Emil Walsted, ${ }^{2,3}$ James H Hull ${ }^{1,2}$}

1 Lung Function Unit at the Royal Brompton and Harefield NHS Foundation Trust, London, UK

${ }^{2} \mathrm{NIHR}$ Respiratory Biomedical Research Unit at the Royal Brompton, Harefield NHS Foundation Trust and Imperial College, London, UK

${ }^{3}$ Respiratory Research Unit, Bispebjerg University Hospital, Copenhagen, Denmark

Correspondence to Dr James H Hull, Lung Function Department, RBH NHS Foundation Trust, London SW3 6HP, UK; j.hull@rbht.nhs.uk, j.hull@rbht.nhs.uk

Acknowledgements We wish to thank the Lung Function Department Staff at the Royal Brompton Hospital for their assistance.

Contributors All authors contributed to the design of the study, analysis of data and preparation of the final manuscript. JHH conceived the idea and acts as guarantor of the paper, taking responsibility for the integrity of the work as a whole, from inception to published article.

\section{Competing interests None declared.}

Provenance and peer review Not commissioned; externally peer reviewed.

(c) Article author(s) (or their employer(s) unless otherwise stated in the text of the article) 2018. All rights reserved. No commercial use is permitted unless otherwise expressly granted.

\section{Check for updates}

To cite Roberts C, Ward S, Walsted E, et al. Thorax 2018;73:385-387.

Received 10 March 2017

Revised 2 May 2017

Accepted 15 May 2017

Published Online First 29 June 2017

Thorax 2018:73:385-387.

doi:10.1136/thoraxjnl-2017-210246

\section{REFERENCES}

1 Butterfield K, Association for Respiratory Technology and Physiology. ARTP Survey 2005 - ARTP Reports. N.p., 1 Mar 2006, Web 17 Jan 2017.

2 Weissman C. Pulmonary function after cardiac and thoracic surgery. Anesth Analg 1999:88:1272-9.

3 Cooper BG. An update on contraindications for lung function testing. Thorax 2011:66:714-23.

4 Enright PL, Lebowitz MD, Cockroft DW. Physiologic measures: pulmonary function tests. Am J Respir Crit Care Med 1994;149:59-18.

5 Montenegro HD, Chester EH, Jones PK. Cardiac arrhythmias during routine tests of pulmonary function in patients with chronic obstruction of airways. Chest 1978;73:133-9.

6 Contraindication to use of spirometry. AARC clinical practice guidelines spirometry, 1996 Update. Respir Care 1996:41:639-36.

7 Newman BH. Vasovagal reaction rates and body weight: findings in high- and low-risk populations. Transfusion 2003:43:1084-8

8 Keteyian SJ, Isaac D, Thadani U, et al. Safety of symptom-limited cardiopulmonary exercise testing in patients with chronic heart failure due to severe left ventricular systolic dysfunction. Am Heart J 2009:158:572-7.

9 Myers J, Arena R, Franklin B, et al. Recommendations for clinical exercise laboratories: a scientific statement from the american heart association. Circulation 2009:119:3144-61. 